\title{
Per Bjørn HALVORSEN, Saint Dominique : du cœur aux frontières de l'Église
}

Johnny Grandjean Gsig Jakobsen

\section{(2) OpenEdition}

\section{Journals}

Édition électronique

URL : https://journals.openedition.org/ccm/5172

DOI : $10.4000 / \mathrm{ccm} .5172$

ISSN : 2119-1026

\section{Éditeur}

Centre d'études supérieures de civilisation médiévale/Université de Poitiers

\section{Édition imprimée}

Date de publication : 1 septembre 2020

Pagination : 202-204

ISBN : 978-2-490783-06-9

ISSN : 0007-9731

Référence électronique

Johnny Grandjean Gsig Jakobsen, «Per Bjørn halvorsen, Saint Dominique : du cœur aux frontières de l'Église », Cahiers de civilisation médiévale [En ligne], 250-251 | 2020, mis en ligne le 01 septembre 2021, consulté le 26 novembre 2022. URL : http://journals.openedition.org/ccm/5172 ; DOI : https://doi.org/ $10.4000 / \mathrm{ccm} .5172$

\section{(c) $)(1) \Theta$}

Creative Commons - Attribution - Pas d'Utilisation Commerciale - Pas de Modification 4.0 International - CC BY-NC-ND 4.0

https://creativecommons.org/licenses/by-nc-nd/4.0/ 
Per Bjørn Halvorsen, Saint Dominique : du coeur aux frontières de l'Église, A. Porret et T. Patfoort (trad.), P. RAfFin (préf.), Paris, Éditions du Cerf (L’histoire à vif), 2011.

En 1216, le chanoine espagnol Dominique de Guzman (v. 1175-1221, que la postérité connait mieux sous le nom de saint Dominique) fonda un nouvel ordre monastique : l'Ordre des Frères Prêcheurs. Au cours $\mathrm{du} \mathrm{XIII}^{\mathrm{e}} \mathrm{s}$. et en marge du christianisme, des couvents de l'Ordre ont été établis dans toute l'Europe, où les frères de Dominique ont joué un rôle important dans l'enracinement de la compréhension et de la croyance chrétiennes. Bien que loin d'être aussi personnellement intriguant et spirituellement attrayant pour l'érudit moderne que son contemporain, saint François, aussi fondateur d'un Ordre, il y a tout de même de nombreuses biographies sur saint Dominique. Ce nouvel ouvrage s'intéresse aux relations de saint Dominique avec la Scandinavie. Une telle approche est un choix plutôt difficile à traiter pour un livre entier car seules quelques lignes préservées dans des sources médiévales relient le saint au Nord; Nord qui fait toujours l'objet de débats à savoir s'il s'agit bel et bien de la Scandinavie. C'est dans ce contexte que l'a., Per Bjørn Halvorsen (1939-2007), s'était en effet lancé en 2002 dans sa biographie, Dominikus En europeers liv på 1200-tallet [Dominique - La vie d'un Européen au XIII ${ }^{\mathrm{e}}$ siècle] (Per Bjørn Halvorsen, Dominikus - En europeers liv på 1200-tallet, Oslo, Novus forlag [Institutt for sammenliknende kulturforskning. Serie B, 111], 2002), dans sa langue maternelle, en norvégien, et dont l'œuvre présente est une traduction posthume en français. Cependant, si P. B. Halvorsen a réussi à écrire 296 p. dans la 
version originale et $355 \mathrm{p}$. dans la nouvelle traduction sur ces quelques lignes manuscrites, c'est en partie parce qu'il a délibérément décidé de mettre en ouvre son voyage biographique comme un voyage fait de détours. Principalement dans la version originale norvégienne, ce parcours, qui change constamment de direction, laisse le lecteur dans l'expectative et le rend même parfois dubitatif quant au réel sujet du livre. Est-ce sur saint Dominique en général? Est-ce sur ses (possibles) connexions avec la Scandinavie? Sur les débuts de l'histoire de l'Ordre des Prêcheurs? Ou sur les débuts de l'Ordre en Scandinavie? La vérité, c'est que le livre de P. B. Halvorsen est sur tout cela à la fois, étayant les sous-thèmes individuels avec des descriptions assez approfondies de leurs éventuels contextes et des environnements dans lesquels ils existaient. Dans la version originale norvégienne, ces descriptions atteignent souvent un degré montrant peu de relation directe avec saint Dominique ou son Ordre.

Si le lecteur peut faire abstraction du thème de l'errance, c'est en effet un très bon livre, lisible et intéressant, offrant une bonne vision et une possible compréhension des motifs de Dominique avec l'Ordre depuis le jour où il a eu l'idée même de le créer, comment il a été formé au moment du quatrième concile de Latran (1215), comment l'Ordre s'est développé en tant qu'institution dans la génération suivant sa mort, et non des moindres, comment les Frères Prêcheurs ont été introduits et se sont établis en Scandinavie au cours de la première moitié du $\mathrm{XIII}^{\mathrm{e}} \mathrm{s}$. Mieux que n'importe quelle œuvre avant la sienne, le livre original de P. B. Halvorsen présente les débuts de l'histoire et les idées de l'Ordre dominicain et de ses relations avec la Scandinavie à un large public scandinave. Mieux que tout ouvrage avant celui-ci, la nouvelle traduction en français présente les points de contact possibles entre saint Dominique et la Scandinavie, ainsi que les débuts de l'Ordre scandinave.

Pour une perspective savante, le livre s'engage particulièrement sur deux questions majeures qui ont longtemps été débattues dans l'histoire dominicaine. Tout d'abord, P. B. Halvorsen reprend une vieille discussion sur la question de savoir si les voyages de Dominique vers les Marchiis l'ont amené au Danemark ou lui ont fait faire d'autres avancées aux frontières de l'Europe, question sur laquelle l'a. se range clairement du côté de Jarl Gallén (ID., La Province de Dacie de l'ordre des Frères prêcheurs, 1 : Histoire générale jusqu'au Grand Schisme, Helsinki/Rome, Institutum Historicum FF. Praedicatorum [Dissertationes Historicae, 12], 1946), par ex., qui est en faveur de l'interprétation danoise contrairement aux avis de Vladimir J. Koudelka (ID., « Notes pour servir à l'histoire de Saint Dominique [Part II] ",Archivum Fratrum Praedicatorum, 43, 1973, p. 5-27) et Simon Tugwell (ID., « Notes on the Life of St Dominic ", Archivum Fratrum Praedicatorum, 68, 1998, p. 5-116). En relation étroite avec ce sujet, on trouve la discussion sur " Le mythe nordique » vs « Le mythe Couman »: l'évêque Diego et Dominique ont-ils vraiment demandé au pape Innocent IV la permission d'être démis de leurs fonctions afin de s'impliquer dans leur mission parmi les Coumans comme indiqué dans une chronique dominicaine, ou s'agit-il d'une erreur ultérieure d'interprétation de leur souhait réel de prendre part à la croisade danoise contre les païens baltes? Ici, P. B. Halvorsen rejoint Marie-Humbert Vicaire (ID., Histoire de saint Dominique, Paris, Éditions du Cerf, 1957 [2 éd. : Paris, Cerf [Semeurs], 1982]) et J. Gallén en croyant ce dernier, contre les interprétations de $\mathrm{S}$. Tugwell et $\mathrm{V}$. Koudelka. Deuxièmement, P. B. Halvorsen traite également de la question complexe et controversée de savoir quand et comment les 12 premières provinces de l'Ordre dominicain ont été établies, offrant une assez bonne explication en faveur de la thèse de $\mathrm{S}$. Tugwel (peut-être même encore plus claire que celle de S. Tugwell lui-même : Simon Tugwell, "The Evolution of Dominican Structures of Government II: The First Dominican Provinces ", Archivum Fratrum Praedicatorum, 70, 2000, p. 5-109). Enfin, il convient de souligner que le livre comprend une version récente du texte édité de la chronique Historia ordinis prcedicatorum in Dania, à la fois en latin et en traduction française, et, peut-être plus important encore, elle est accompagnée d'un appareil critique exhaustif et exceptionnel, avec commentaires, explications et discussions sur pratiquement tous les éléments du texte.

Comme cette revue concerne la traduction du livre original de P. B. Halvorsen de 2002, traduit par Agnès Porret et Thomas Patfoort, une comparaison des deux versions est en cours. À mon avis, pour la plupart des lecteurs, la traduction est une version très améliorée puisque plusieurs des " détours 》 les plus longs et moins évidents du texte source en norvégien ont été judicieusement laissés de côté par les éditeurs français. Ainsi, le récit de la traduction semble beaucoup plus cohérent. En ce qui concerne les illustrations : une quinzaine de nouvelles photos ont été incluses, principalement du nord de l'Espagne et du sud de la France, ainsi que quelques photographies récentes de la maison et de l'église dominicaines dans l'actuelle Oslo. En contrepartie, une poignée de photographies des vestiges architecturaux médiévaux 
de Scandinavie ont été laissée de côté, ce qui est dommage. Enfin, la traduction française comprend une préface de Pierre Raffin o.p., évêque de Metz, et une postface des sœurs dominicaines d'Oslo.

Dans l'ensemble, le livre de P. B. Halvorsen sur saint Dominique, et surtout cette nouvelle traduction française, est une lecture très recommandable pour tous ceux qui s'intéressent à l'histoire dominicaine en particulier et à l'histoire religieuse de l'Europe du Nord du XIII ${ }^{\mathrm{e}} \mathrm{s}$. en général.

Johnny GrandJEAn Gsig JaKobSen Université de Copenhague, Danemark 